\title{
Serial processing in melody identification and the organization of musical semantic memory
}

\author{
MATTHEW D. SCHULKIND \\ Amherst College, Amherst, Massachusetts
}

\begin{abstract}
Unlike the visual stimuli used in most object identification experiments, melodies are organized temporally rather than spatially. Therefore, they may be particularly sensitive to manipulations of the order in which information is revealed. Two experiments examined whether the initial elements of a melody are differentially important for identification. Initial exposures to impoverished versions of a melody significantly decreased subsequent identification, especially when the early exposures did not include the initial notes of the melody. Analyses of the initial notes indicated that they are differentially important for melody identification because they help the listener detect the overall structure of the melody. Confusion errors tended to be songs that either were drawn from the same genre or shared similar phrasing. These data indicate that conceptual processing influences melody identification, that phrase-level information is used to organize melodies in semantic memory, and that phrase-level information is required to effectively search semantic memory.
\end{abstract}

Most theories of object identification are based on evidence gathered either from visual or verbal stimuli. These theories generally do not consider musical stimuli, although melodies are frequently encountered and easily identified both inside and outside of the laboratory (Bartlett \& Snelus, 1980; Cuddy, 1993; Hébert \& Peretz, 1997; Schulkind, Hennis, \& Rubin, 1999; White, 1960; Yalch, 1991). Thus, additional research and theorizing about melody identification is justified because of its relevance to real-world behavior. In addition, research on melody identification is of theoretical interest because it may shed light on the generalizability of extant theories and may broaden our conception of the processes involved in object identification. In fact, several lines of evidence suggest that melody identification is not exactly analogous to identification of the kinds of objects traditionally studied in the literature. For example, whereas presenting a subject with the name of a distractor does not influence the identification of line drawings (Snodgrass \& Hirshman, 1991), such a manipulation reliably decreases the identification of familiar melodies (Schulkind, 2002). Furthermore, lateral competition - interference caused by nontarget foils (e.g., knowledge of "dog" interferes with identifying "cat")-is a central component of most theories of visual object and spoken word identification (Jusczyk \& Luce, 2002) but does not appear to play a role in melody identification (Schulkind, 2004).

Although the failure to observe effects of lateral competition is at odds with most theories of visual object iden-

I thank David Rubin and Tamara Rahhal, as well as Mari Jones and three anonymous reviewers, for helpful comments on an earlier draft. Correspondence concerning this article should be addressed to M. D. Schulkind, Department of Psychology, Amherst College, Amherst, MA 01002 (e-mail: mdschulkind@amherst.edu). tification, it is consistent with the cohort model of spoken word identification (Marslen-Wilson, 1987). Interestingly, spoken words and melodies share one feature that is atypical of many of the kinds of stimuli that are used in the visual object identification literature: Whereas visual objects are primarily spatial stimuli, spoken words and melodies are primarily temporal stimuli. Melodies and spoken words have temporal beginnings and endings that are not arbitrary; in addition, the serial order and timing of elements within the stimulus are intrinsic to its identity. Thus, one might hypothesize that the processes involved in spoken word and melody identification would bear similarities to each other and differences from those associated with visual object recognition.

The present experiments were designed to explore similarities between melody and spoken word identification. In particular, the experiments evaluated whether a central tenet of the cohort model of spoken word identificationthat initial segments of a word are vital for identification-would be observed with melodic stimuli. Briefly, the cohort model claims that words are identified primarily via bottom-up processes. The early perceptual information activates a set of potential items that share the initial segments; for example, "bl" would activate "blue," "bland," "blade," and so on. Although several lines of evidence support the idea that word onsets are vital for identification (e.g., Marslen-Wilson \& Zwitserlood, 1989; Warren \& Marslen-Wilson, 1987, 1988), evidence does not support the most stringent claim that identification cannot occur if initial phonemes are ambiguous or erroneous (Marslen-Wilson, Moss, \& van Halen, 1996). It should also be noted that no other prominent theory of spoken word identification confers any special status on the initial phonemes of a word (see Jusczyk \& Luce, 2002, for a review). 
This particular aspect of the cohort model was selected because it fits nicely with the literature on melody perception. Data suggest that the beginning of a melody is where the global organization of a melody (e.g., key, meter, rhythm) is established (Butler, 1992; Krumhansl, 1979, 1990). Several theories of music perception suggest that listeners use musical information to generate expectancies about what is likely to follow (Jones \& Boltz, 1989; Narmour, 1990). If the initial notes of the melody are closely associated with the prevailing key, meter, and/or rhythm, listeners' expectations are more likely to be confirmed, which should facilitate both melody perception and subsequent identification. Conversely, if the initial notes are more distantly associated with the global organization of the piece, perception and identification should suffer. This explanation-which will be referred to as the structural centrality/expectation hypothesis-predicts that, relative to other notes in the melody, the initial notes will be more likely to include tones closely associated with the key and/or will include longer, more metrically accented notes. An alternative explanation-which will be referred to as the serial organization/search hypothesis - holds that initial notes are important because of the way the mental lexicon for melodies is organized and searched. Searching the musical lexicon may be accomplished by scanning the region of mental space that contains melodies beginning with a particular note, interval, or phrase, and narrowing the set of contenders by matching subsequent segments with those stored in memory. As an analogy, consider looking up a word in a standard dictionary. First, one flips to the pages for the initial letter of the word, and then continues to scan the pages for the second and third letters and so on until the word is located. According to this explanation, listeners need the initial notes of the melody to locate its representation in the musical lexicon.

Despite their differences, both the structural centrality/ expectation and the serial organization/search hypotheses predict that manipulations occurring at the beginning of a melody should have a more significant effect on identification than manipulations occurring later in the melody. Some data in the literature are consistent with this claim. Hébert and Peretz (1997) found that matching a title to a familiar melody was easier when the title was presented early in the trial. This result only obtained for altered versions of a target melody that included either the rhythmic or pitch pattern, but not both. Intact melodies did not show any effect of the onset/offset manipulation, although this result is difficult to interpret because performance was at ceiling $(\approx 95 \%$ correct). A different result might be obtained for intact melodies identified under more challenging conditions.

The present study evaluated the significance of initial segments for melody identification using the partial exposure interference (PEI) paradigm (Schulkind, 2004). Briefly, the subjects' task was to identify highly familiar melodies based on a limited number of notes. The notes were revealed incrementally across several trials and were always presented in the correct serial order. Different conditions varied the way information was incremented such that the first notes of the melody were not always the first notes heard by the subject (see Figure 1). However, across all conditions, the final exposure for each song was identical, consisting of the first several notes of the melody. The primary question was whether identification performance would be affected by the order in which the information was revealed. An affirmative result would indicate that the beginnings of melodies are crucial for melody identification. Assuming this result will obtain, the next logical question was why initial notes are differentially important. The structural centrality/expectation and serial organization/search hypotheses were evaluated by analyzing the initial elements of the melodies and determining their relationship to identification performance. Finally, a confusion error analysis was conducted to determine whether erroneous responses would reveal anything about the nature of musical semantic memory.

\begin{tabular}{|c|c|c|c|}
\hline Condition & 1st Exposure & 2nd Exposure & 3rd Exposure \\
\hline Fixed & 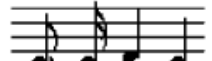 & & \\
\hline Forward & & $\Rightarrow$ & \\
\hline Random & 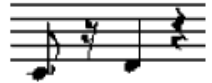 & $\overline{\equiv y}$ & $\Rightarrow$ \\
\hline Backward & 声 & $\overline{F+1}$ & 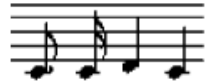 \\
\hline
\end{tabular}

Figure 1. Examples of the fixed, forward, random (Experiments 1 and 2), and backward (Experiment 2) conditions using the melody for "Happy Birthday to You." 


\section{EXPERIMENT 1}

PEI refers to the fact that initial exposures to a degraded version of a target inhibit subsequent identification of that object. This effect is quite general, having been observed with photographic images (Bruner \& Potter, 1964), line drawings (Lindfield \& Wingfield, 1999; Lindfield, Wingfield, \& Bowles, 1994; Luo \& Snodgrass, 1994; Schulkind, 2000; Snodgrass \& Hirshman, 1991), visually presented words (Peynircioğlu, 1987, 1990; Peynircioğlu \& Watkins, 1986), aurally presented words (Gibson \& Watkins, 1991), and aurally presented familiar melodies (Schulkind, 2000, 2002, 2004). Most experiments compare performance across two conditions, labeled fixed and ascending. The fixed condition consists of one exposure to a moderately degraded version of an object; with melodies, the degradation typically involves restricting the number of notes the listener hears. The ascending condition consists of several exposures. Starting with a highly degraded version (i.e., one that includes only the first two notes of the melody), notes are appended over time until the moderately degraded version in the fixed condition is reached. Typically, performance in the fixed condition is superior to performance in the ascending condition, even though both methods ultimately present the same information.

The first experiment included three conditions. The fixed condition consisted of the first several notes of a familiar melody; for example, the subjects heard the first four notes of "Happy Birthday to You" (see Figure 1). In addition, two types of ascending presentation formats were used. Both of the ascending conditions consisted of multiple exposures to the target. The first exposure consisted of two notes; each subsequent exposure added one note until the number of notes in the fixed condition was reached. Thus, the final exposure in both of the ascending conditions was identical to the single exposure in the fixed condition. The two ascending conditions differed in terms of which notes were presented for each trial. The forward condition was identical to what has been called ascending presentation in previous studies (Schulkind, 2000, 2002, 2004); the first exposure consisted of the first two notes of the melody; the second consisted of the first three notes, and so on (see Figure 1). As its name implies, the notes heard in the random ascending condition were selected randomly, starting with two notes, and adding a new randomly selected note at each successive exposure; silent intervals replaced the notes that were not selected (see Figure 1).

The fixed condition was expected to yield better performance than the forward condition, which would replicate previous work using musical stimuli. The fixed condition was also expected to yield better performance than the random condition. Although the random condition has not been used in previous experiments with music, similar findings have been observed with verbal stimuli (Gibson $\&$ Watkins, 1991). The relative performance of the forward and random conditions was more difficult to predict, but the result should provide important information regarding the relative importance of initial notes for melody identification. If the forward and random conditions yielded similar levels of performance, one would be able to conclude that the initial features of a melody are no more important for identification than are subsequent features. Alternatively, a significant advantage in the forward condition relative to the random condition would indicate that initial features are crucial for melody identification.

The implementation of the random condition also allows another important theoretical issue to be addressed. Previous research has demonstrated that some manipulations affect PEI with melodies but do not affect PEI with visual stimuli. For example, whereas distractor song titles reduce melody identification (Schulkind, 2002), distractor object names do not affect the identification of line drawings (Snodgrass \& Hirshman, 1991). One explanation for these cross-class differences is methodological: Researchers typically adapt the basic PEI paradigm to suit the stimulus class in their experiments. For example, in Snodgrass and Hirshman's research with line drawings, the difference between the fixed and ascending conditions was the number of pixels deleted from the drawing. For visually presented verbal stimuli (e.g., Peynircioğlu \& Watkins, 1986), the fixed and ascending conditions differed in terms of the number of letters deleted from the word, whereas for aurally presented verbal stimuli, the fixed and ascending conditions differed in terms of the number of $100-\mathrm{msec}$ silent intervals introduced into the word (e.g., Gibson \& Watkins, 1991). Although different from one another, each of these methodologies is similar in that the target was degraded by introducing spatial or temporal gaps into the stimulus. The methodology used in previous research with music was quite different in that the ascending condition (which corresponds to the forward condition described above) did not degrade the stimulus by introducing silent intervals within the stimulus. Thus, the random condition employed in Experiment 1, which includes silent intervals, will close the methodological "gap" between research in melodies and research using other stimuli. If the random condition produces a significant PEI effect, one would conclude that differences between musical and visual stimuli are not a result of the methodology used to degrade the stimulus.

\section{Method}

Subjects. Twenty-seven undergraduate volunteers received partial credit toward a course requirement in exchange for their participation. No subjects were music majors; on average, the subjects had formally studied $1.0(S D=0.7)$ instrument for an average of 6.6 $(S D=5.8)$ years.

Materials. The 33 songs used in the experiment, selected from a set of 34 songs used in previous research (Schulkind, 2000), were pretested by a group of 97 undergraduate subjects to ensure their familiarity. The songs were drawn from one of the following categories (with a representative tune appearing in parentheses): patriotic ("Yankee Doodle"); Christmas/religious ("Frosty the Snowman"); pop/folk ("Yesterday"); children's ("Pop! Goes the Weasel"); traditional ("Happy Birthday to You"); and movies ("Do Re Mi"). A separate group of subjects identified the target stimuli in an ascending sequence in which they were allowed to hear as many as 24 notes; mean percent correct was $95.5 \%(S D=6.5)$. Thus, these 
songs were easily identified when the subjects were given sufficient information.

A sheet music arrangement of each song was obtained and transferred to a Macintosh IIsi computer using the Songworks software package (Ars Nova). The stimuli incorporated a wide range of keys (e.g., C, G, F, Eb, Ab), as well as both simple (2/4, 3/4, 4/4) and complex meters (6/8); all stimuli were played with a piano timbre. A subset of the notes from each song was selected by determining the number of notes that led to moderate identification performance $(40 \%-65 \%)$ in past research (Schulkind, 2000). For example, the subjects heard the first four notes of "Happy Birthday to You." In the fixed condition, the subjects received one exposure consisting of all the notes for that song (e.g., the first four notes of "Happy Birthday to You"; see Figure 1). On average, the fixed condition presented 6.0 notes $(S D=1.8)$. The tempo of each melody was adjusted to sound "natural" $(M=117.62$ quarter notes per minute, $S D=26.83$, range 75-150); for each song, the tempo was held constant across all conditions.

Each trial in the forward and random conditions consisted of multiple exposures to the target melody. The first exposure consisted of two notes. Each subsequent exposure added one more note: the second exposure consisted of three notes, the third exposure consisted of four notes, and so on. Successive exposures were separated by a period of silence equal to one eighth-note. Because the tempi of the song varied, the silent duration lasted between 180 and $320 \mathrm{msec}$. This interval was well within the range needed to observe PEI (Schulkind, 2000) and prevented the subjects from making a response until hearing the final exposure in the sequence. In the forward condition, the first exposure consisted of the first two notes, with each successive exposure adding the following note (i.e., the second exposure consisted of the first three notes, the third exposure consisted of the first four notes, etc.). Notes were added one at a time until the specified number of notes in the fixed condition for that song was reached. In the random condition, the first exposure consisted of two randomly selected notes. The remaining notes were replaced by silent intervals equal to the duration of the missing notes. Additional notes were selected randomly without replacement and added one at a time until the specified number of notes in the fixed condition for that song was reached. Thus, the final exposures in both the forward and random conditions presented the same information as the one and only exposure in the fixed condition.

Procedure. At the start of the experiment, the three presentation conditions (fixed, forward, and random) were described and the subject heard an example of all three styles using the melody for "Old McDonald Had a Farm." Every subject heard the stimulus songs in the same random order. Three counterbalance orders were created such that, across the experiment, each song appeared an equal number of times in each condition.

The subjects were instructed to give one of the following five responses after each trial: (1) "I have no idea"; (2) "It sounds familiar but I am not certain enough to make a guess"; (3) "I am not sure but it might be "; (4) "I am pretty sure that it is "; or (5) "I am sure that it is__." The five response options were printed on a sheet of paper placed on the desk in front of the subject. A response was considered correct if it included the correct title (or at least five words from the target song) regardless of the confidence rating. Analyses of the confidence ratings data perfectly matched those of the percent correct data and thus, will not be discussed further. The subjects controlled the pace of the experiment, although they were required to respond within $10 \mathrm{sec}$ of the end of a given trial; the experimenter monitored the response period with a hand-held stopwatch. The subjects were not told the title of a song that they failed to identify.

At the end of the experiment, the subjects were presented with a sheet of paper listing the titles of all of the songs used in the experiment interspersed among 27 song titles that were not part of the experiment. The subjects rated their familiarity with the songs on a 5-point scale ranging from "I know this song extremely well" to "I have never heard this song before today." Data for songs that subjects did not know $(<2.5 \%)$ were eliminated from all subsequent data analyses.

\section{Results}

All analyses reported in this article were conducted using both items and subjects as the units of analysis. Because all analyses revealed the same pattern of results, only those using the subject data will be reported. When appropriate, the degrees of freedom were adjusted using the Greenhouse-Geisser procedure; all reported degrees of freedom and mean square errors represent the unadjusted values. Alpha was set at .05 for all analyses.

Mean percent correct identification for the fixed, forward, random, and backward conditions are presented in Figure 2. The data were submitted to a one-way withinsubjects analysis of variance (ANOVA) with presentation style (fixed, forward, random, backward) as the independent variable. The analyses yielded a main effect of presentation style $\left[F(2,52)=11.243, M S_{\mathrm{e}}=.029\right]$. Tukey's honestly significant difference (HSD) was calculated to determine which levels of the independent variable differed from one another; depending on the specific pairs tested, Tukey's HSD varied between .09 and .10. The results of this analysis indicated that all three conditions were significantly different from one another.

Correlation analyses were conducted to determine whether there was a relationship between the two measures of musical experience (years of musical training and number of instruments played) and identification performance. Significant correlations were observed between both measures of musical experience and performance in the forward and random conditions [all $r \mathrm{~s}(26)>.40$, all $p$ s $<.05$ ]; no other correlations were significant. The significant correlations are of little concern because the crucial comparisons in the current research were the differences between conditions. Neither measure of musical experience yielded significant correlations with any of the intercondition difference scores [all $r \mathrm{~s}(26)<.28$, all $p \mathrm{~s}>$ .15]. This result is not uncommon because the use of familiar melodies often decreases or eliminates expertise differences (Schulkind, Shulman, \& Rubin, 2003; Smith, Kemler Nelson, \& Appleton, 1994).

\section{Discussion}

As predicted, both ascending conditions (random and forward) yielded lower identification performance than the fixed condition. This represents both a replication and extension of the PEI effect in musical stimuli. Inserting temporal gaps into an ascending sequence did not eliminate the PEI effect observed in previous work using forward presentation. In fact, the magnitude of the PEI effect was significantly larger when the fixed condition was compared with the random condition than when the fixed condition was compared with the forward condition. This indicates that any empirical differences observed between PEI effects in music and other stimuli are unlikely to have resulted from differences in methodology. Instead, the 


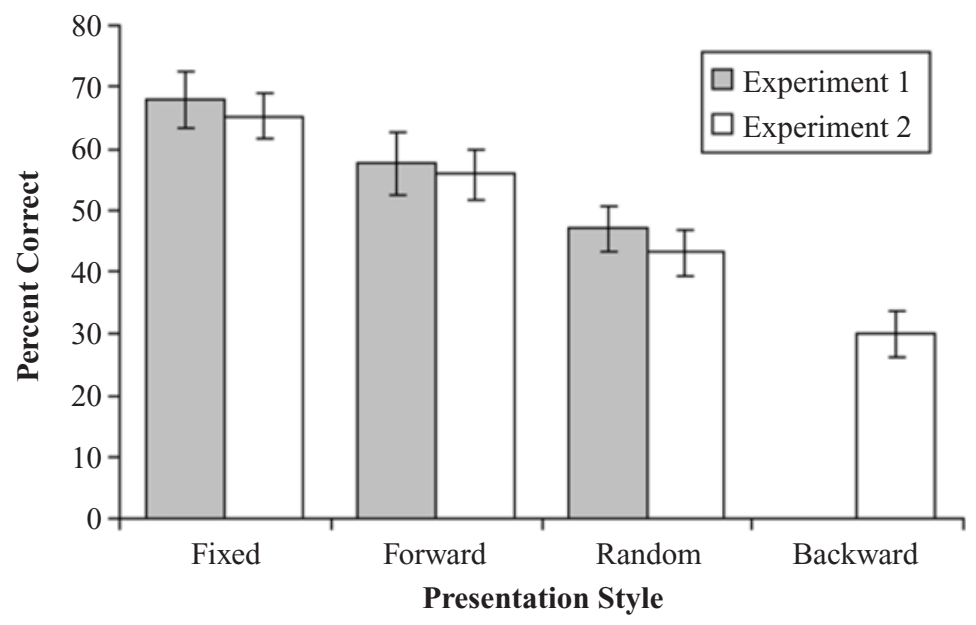

Figure 2. Mean percent correct identification by presentation style across Experiments 1 and 2.

data suggest that the disparities observed in the literature reflect differences in the way melodies and visual objects are identified.

More importantly, the data support the hypothesis that initial segments of a melody are crucial for identification. The random condition, which obscured the identity of the initial notes, yielded identification performance that was significantly worse than performance in the forward condition, which consistently presented the initial notes of the melody first. However, two alternative explanations can account for the observed differences between the forward and random conditions. First, the impoverished, early exposures in the random condition were greater in duration than the corresponding exposures in the forward condition. This occurred because the deleted notes were replaced by silent intervals (see Figure 1). Previous research has shown an inverse relationship between identification performance and the length of exposure to the degraded versions of the target (Bruner \& Potter, 1964; Luo \& Snodgrass, 1994). Second, in the forward condition, silent intervals unambiguously signaled that a new exposure to the melody was about to begin. This contingency was not duplicated in the random condition because some silent intervals represented deleted notes. Thus, the observed performance decrement in the random condition may have resulted from an inability to distinguish between successive exposures. During postexperimental interviews, the subjects were asked whether parsing the stimuli in the forward and random conditions had been difficult. Although no subject reported any such difficulty, this possibility will be examined more closely in Experiment 2.

\section{EXPERIMENT 2}

Experiment 2 was designed to provide an unambiguous demonstration of the importance of initial information for melody identification. This goal was realized by intro- ducing an ascending presentation format that obscured the identity of the initial notes in the melody while avoiding the problems associated with the random condition from Experiment 1. To that end, Experiment 2 included a third ascending condition that will be referred to as the backward condition. Like the two ascending conditions used in Experiment 1, the backward condition consisted of multiple exposures to the target in which the amount of information was incremented one note at a time. However, instead of starting with the first two notes of the melody, the backward condition began with the last two notes presented in the fixed condition. For example, in the backward condition of "Happy Birthday to You" (see Figure 1), subjects initially heard notes 3 and 4, followed by an eighth-note rest. In the second exposure, they heard notes 2,3 , and 4 , followed by an eighth-note rest. In the third exposure, they heard all four notes given in the fixed condition. It is important to remember that the notes were always heard in the correct serial order (i.e., note 2, followed by note 3 , followed by note 4 ); the "backward" label reflected the fact that the notes were added from the end to the beginning.

Because the backward condition did not replace notes with silent intervals, the exposure duration in this condition was comparable to that of the forward condition; and the interval between exposures was identical. Furthermore, a silent interval always signaled that a new exposure to the stimulus was about to begin. Thus, the forward and backward conditions differed only in terms of the order in which information was revealed. A significant difference between these conditions would confirm that initial segments of a song are particularly important for melody identification.

\section{Method}

Subjects. Thirty-six undergraduate volunteers received partial credit toward a course requirement in exchange for their participation. No subjects were music majors; on average, the subjects had 
formally studied $1.1(S D=1.1)$ instruments for an average of 3.7 $(S D=4.2)$ years

Materials. The base stimuli and the fixed, forward, and random conditions were the same as those used in Experiment 1. In the backward condition, the first exposure consisted of the last two notes presented in the fixed condition (e.g., notes 3 and 4 for "Happy Birthday to You"). Subsequent exposures added the next earliest notes (e.g., notes 2-4), until the number of notes matched that in the fixed condition. Successive exposures were separated by a silent interval equal to one eighth-note. Four counterbalance orders were constructed so that each song appeared once in each presentation style. This necessitated using only 32 of the 33 songs used in Experiment 1 ("Dixie" was eliminated because it had been rated the least familiar song in Experiment 1).

Procedure. The same procedure was used as in Experiment 1 . Data for songs that subjects claimed not to know (less than $1 \%$ of the data) were not analyzed.

\section{Results}

Mean percent correct identification for fixed, forward, random, and backward presentations are presented in Figure 2. The data were submitted to a one-way withinsubjects ANOVA with presentation style (fixed, forward, random, backward) as the independent variable. The analyses yielded a main effect of presentation style $[F(3,105)=$ $\left.37.965, M S_{\mathrm{e}}=.022\right]$. Tukey's HSD was calculated to determine which levels of the independent variable differed from one another; depending on the specific pairs tested, Tukey's HSD varied between .06 and .08 . The results of this analysis indicated that all four conditions were significantly different from one another.

As in Experiment 1, there were some significant correlations between musical experience and performance. The years of training measure was significantly correlated with performance in the fixed $[r(35)=.51, p<.01]$ and random $[r(35)<.36, p>.05]$ conditions; no other correlations were significant. However, as in Experiment 1, the expertise measures were not correlated with the differences between conditions [all $r \mathrm{~s}(35)<.11$, all $p \mathrm{~s}>.50$ ].

The performance decrement in the backward condition relative to the fixed condition provides clear evidence of the importance of initial notes in the identification of familiar melodies. However, this result does not address why initial notes are important. Two hypotheses were laid out in the introduction. The structural centrality/expectation hypothesis holds that notes at the beginning of a melody are differentially important for highlighting the global structure of a piece. This hypothesis predicts that the initial notes in the melody should be structurally more important than the initial notes heard in the backward condition, which were selected more or less at random. That is, they should be more closely associated with the tonality of the piece and should be more strongly accented rhythmically and/or metrically. The serial organization/search hypothesis holds that initial notes are important because musical semantic memory is organized according to the initial notes of melodies. This hypothesis predicts that identification performance in the backward condition should vary as a function of the relationship between the initial notes in the backward condition and the initial notes in the melody. Specifically, the more different the initial notes in the backward condition are from the initial notes in the melody, the worse identification performance ought to be.

Structural centrality/expectation. The structural centrality/expectation hypothesis was evaluated by coding the first two notes of the melody and the first two notes of the backward condition using several components of the coding scheme adopted by Schulkind et al. (2003). The tonal function (Krumhansl, 1979, 1990) of each note was placed into one of six classes that were coded in decreasing order of centrality to the tonal center using the integers from 6 to 1 ; the six categories were: tonic (the 1st note in the scale; e.g., $\mathrm{C}$ in $\mathrm{C}$ major), dominant (the 5 th note in the scale; e.g., $\mathrm{G}$ in $\mathrm{C}$ major), mediant (the 3rd note in the scale; e.g., $\mathrm{E}$ in $\mathrm{C}$ major), leading tone (the 7th note in the scale, which immediately precedes the return to the tonic; e.g., $\mathrm{B}$ in $\mathrm{C}$ major), other diatonic tones (other notes within the key; e.g., D, F, and $\mathrm{A}$ in $\mathrm{C}$ major), and nondiatonic tones (notes outside of the key; e.g., the sharps and flats for $\mathrm{C}$ major). The temporal characteristics of the initial tones were also coded. Jones and Boltz (1989) argued that relatively long notes serve as anchors that draw the listener's attention and help reveal important structural characteristics of the melody; thus one might expect the initial notes of the melody to be longer. Note duration was coded relative to the beat period for each melody and was placed into three categories: shorter than one beat $(0)$, equal to one beat (1), and greater than one beat (2). Metrical accent was coded in terms of the strength of the placement of the note within the metric hierarchy. The system (Schulkind et al., 2003) distinguished between four classes of accent. In decreasing order of strength, these were (with associated codes in parentheses): 1 st beat of the measure (4), 3rd beat of the measure (3), 2nd or 4th beat of the measure (2), and half-beats (1). The metrical pattern of the first two notes was also coded in terms of the relative strength of the accent of the two notes; the pattern was either from a stronger beat to a weaker beat (SW, coded with a 1) or the reverse (WS, coded with a 0 ). This factor was coded because Dowling, Lung, and Herrbold (1987; see also Dowling, 1974) found that identifying a target interleaved with distractors was easier when the target was played on the odd-numbered tones, which they argued placed them on the implicit beat. Listeners may have expectations regarding metrical accent that would lead to better performance for melodies beginning SW, relative to melodies beginning WS. Finally, the location of each note within the musical phrase was coded because this has been shown to be an important predictor of identification performance (Schulkind et al., 2003). Notes that occurred at a phrase boundary (either the beginning or the end) were coded with a 1; all other notes were coded in terms of their distance from a phrase boundary (i.e., the second and penultimate note in a phrase were coded with a 2 , etc.).

The data and the results of the associated $t$ test are presented in Table 1; the Type I error rate was controlled using Tukey's HSD. The first two notes of the fixed and 
Table 1

Means and Standard Deviations for Measures of the Musical Characteristics of the First Two Notes in the Fixed and Backward Conditions in Experiment 2 Along With the Associated Values of Tukey's HSD

\begin{tabular}{lcccccc}
\hline & \multicolumn{2}{c}{ Fixed } & & \multicolumn{2}{c}{ Backward } & \multirow{2}{*}{ Tukey's HSD } \\
\cline { 2 - 3 } & $M$ & $S D$ & & $M$ & $S D$ & $M$ \\
\hline Tonal function & 4.89 & 0.93 & & 3.80 & 0.96 & $0.49^{*}$ \\
Duration & 0.92 & 0.46 & & 0.97 & 0.44 & 0.17 \\
Metrical accent & 2.73 & 0.46 & & 2.63 & 0.58 & 0.20 \\
Metrical pattern & 0.72 & 0.46 & & 0.47 & 0.51 & $0.24^{*}$ \\
Phrase placement & 3.00 & - & & 4.78 & 2.06 & \\
\hline
\end{tabular}

Note-The standard deviation is missing from the phrase placement variable in the fixed condition because it always included the first and second notes of the song, which were always the first and second notes of the musical phrase. *Significant difference (e.g., difference between the means $>$ Tukey's HSD).

backward conditions differed in terms of tonal function; according to the criteria established in previous work (Krumhansl, 1979, 1990), the initial notes in the fixed condition were more closely associated with the tonality of the piece than were the initial notes in the backward condition. The temporal characteristics of the fixed and backward conditions were more similar. Although the two conditions did not differ in terms of average note duration or average strength of the metrical accent, there was a significant difference in the likelihood of beginning on a relatively strong beat. Almost $75 \%$ of the trials in the fixed condition began with a SW pattern; trials in the backward condition were approximately equally split between SW and WS. Finally, there was a significant difference in the phrase placement of the initial notes in the two conditions. The notes in the fixed condition were located closer to phrase boundaries than were notes in the backward condition. However, the phrase placement data should be interpreted with some caution because the first two notes of the melody were by definition the first two notes of a phrase. Together, these data suggest that the initial notes in a melody are structured differently from those occurring in other serial locations. Relative to the first two notes in the backward condition, the first two notes in the fixed condition were more closely associated with the tonal center, more likely to include a phrase boundary, and more likely to begin on a metrically accented beat. These data support the structural centrality/expectation hypothesis.

Serial organization/search. The serial organization/ search hypothesis predicts that performance in the backward condition will be inversely related to differences between the first two notes in the backward condition and the first two notes in the melody. To control for differences in the ease or difficulty of identifying a particular song, separate analyses were conducted using the fixed and forward conditions as baselines against which to compare performance in the backward condition. These two conditions were selected as baselines because the first two notes in both conditions were also the first two notes in the melody (see Figure 1). Thus, the serial organization/ search hypothesis was evaluated by correlating intracon- dition differences in identification performance (e.g., backward-fixed or backward-forward) with intracondition differences in the musical characteristics (e.g., tonal function, metrical accent pattern, etc.) of the first two notes in the melody and the first two notes in the backward condition. With "Happy Birthday to You" as an example and the fixed condition as the baseline, average percent correct in both the fixed and backward conditions was $78 \%$, so the difference score for identification performance was 0 . The first two notes in the fixed condition of "Happy Birthday to You" were both the dominant (coded with a 5); thus, the average tonal function score in the fixed and forward conditions was 5 . The first note in the backward condition was the dominant (coded with a 5); the second note in the backward condition was a diatonic tone (coded with a 2). Thus, the average tonal function score in the backward condition was 3.5 , and the corresponding difference score for tonal function was $1.5(5.0-3.5)$.

Tonal function, note duration, metrical accent, metrical accent pattern, and phrase placement were coded as described in the previous section. Several other measures were also coded. These additional measures were not included in the analysis of the structural centrality/expectation hypothesis because they could not be classified as more or less central to the organization of a piece in the way that tonal function, duration, and metrical accent could be. The contour direction was coded as either rising (1), falling $(-1)$, or neutral in the case of unisons (0). Although there is no reason to predict that a rising or falling interval would facilitate identification relative to the other, the serial organization/search hypothesis predicts that a rising initial interval in the backward condition would inhibit identification if the initial interval in the melody were falling. Interval size was also examined. We determined whether intervals were the same or different in size, and coded the absolute difference in the size of the interval in semitones.

None of these difference scores were significantly correlated with differences in performance between the fixed and backward conditions. Only the contour direction measure yielded a correlation coefficient that approached significance $[r(31)=.27, p \approx .13]$. As such, a modified contour direction measure was constructed that took into account the ambiguity of unisons, which could form part of a pitch line that was generally rising or falling. For this modified measure, a 0 indicated that both intervals shared the same direction (i.e., up/up, down/down, or unison/unison), a 1 indicated that the two intervals had different contour directions and one of the intervals was a unison (i.e., up/unison, unison/up, down/unison, or unison/down), and a 2 indicated different contours with neither interval being a unison (i.e., up/down or down/up). The modified measure produced a correlation coefficient that was closer to the traditional .05 cutoff but still fell short of significance $[r(31)=.34, p \approx .06]$.

Similar analyses were conducted analyzing differences between the backward and forward conditions. The coding of the initial notes did not change, because the initial 
notes in the forward condition were the same as the initial notes in the fixed condition. However, performance differences were calculated by subtracting mean performance in the backward condition from mean performance in the forward condition. None of the correlations were significant, and the only correlation that approached significance involved the phrase placement variable $[r(31)=$ $.33, p \approx .06]$. Thus, in contrast to the structural centrality/expectation hypothesis, the data suggest that differences between the initial notes of the melody and the initial notes in the backward condition did a very poor job of predicting identification performance in the backward condition.

Confusion error analysis. Across both experiments, the subjects made 929 errors, 846 (91.1\%) of which were errors of omission (i.e., nonresponses). The remaining 83 $(8.9 \%)$ errors were trials for which the subject provided an incorrect title. There were no differences in the number of confusion errors as a function of experimental condition in either Experiment $1\left[F(2,28)=1.51, M S_{\mathrm{e}}=1.27\right]$ or Experiment $2\left[F(3,78)=0.74, M S_{\mathrm{e}}=0.31\right]$.

Although the confusion error rate was low, the incorrect responses did bear interesting relationships to the targets. The Appendix includes a list of all the incorrect titles that were provided in response to each target. One class of error that was immediately apparent was genre confusions; for example, three subjects responded " $\mathrm{Ru}$ dolph the Red-Nosed Reindeer" instead of "Frosty the Snowman." This accounted for 19 of the $83(22.9 \%)$ errors and is in keeping with Halpern's (1984) finding that genre strongly influences similarity ratings for familiar tunes. A second category of errors was labeled phrasing/ meter errors. These errors involved songs that shared the same phrase length and metrical pattern as the target such that the words to one song could easily be sung over the melody of the other, at least through the initial phrase or two. For example, the initial phrases of "Mary Had a Little Lamb" and "Yankee Doodle Went to Town" both consist of seven syllables with accents falling on the oddnumbered syllables. Phrasing/meter errors accounted for 23 of the $83(28.9 \%)$ errors. Taken together, genre and phrasing/meter errors explained just over half of all confusion errors. The remaining errors were difficult to categorize, although 13 involved other songs from the experiment (either targets or "Old McDonald," which was heard during the instructions). Of these errors, $8(9.6 \%)$ involved songs that had been heard earlier in the experiments and thus might be considered episodic confusions based on earlier experimental exposures. Thus, slightly more than $60 \%$ of confusion errors fell into three categories: genre, episodic confusion, and phrasing/meter. The implications of the confusion error analysis will be pursued further in the General Discussion section.

\section{Discussion}

As was the case with Experiment 1, significant differences were obtained between every level of the dependent variable. All three types of ascending presentation (forward, random, backward) led to significant interference, compared with fixed presentation. Furthermore, the way information was presented in the ascending sequences significantly influenced performance. Forward presentation, which preserved the fixed temporal sequencing normally associated with listening to music, resulted in the best performance. Random presentation, which maintained the temporal spacing and ordering of notes, resulted in the next best performance. Backward presentation, which consistently changed the first note that the subjects heard, resulted in the worst performance. These data confirm that initial elements of a melody are differentially important for melody identification.

A comparison of the initial segments of the fixed and backward conditions also provided evidence as to why initial elements are important for identification. The analyses revealed several differences between the musical characteristics of the initial notes of the forward and backward conditions, which is consistent with the structural centrality/expectation hypothesis. In other words, the initial notes of a melody tend to reveal more information about the global structure of the melody than do notes in the middle of the melody. However, none of the differences between the initial notes of the backward condition and the initial notes of the melody (fixed or forward condition) were correlated with intracondition differences in performance. This casts doubt upon the serial organization/search hypothesis.

\section{GENERAL DISCUSSION}

The primary goal of the present study was to determine whether the initial notes of a tune were differentially important for melody identification. The data confirmed that they were. Performance in the random condition was lower than that in either the fixed or forward conditions because the listeners had to wait to hear the initial notes of the melody. The backward condition delayed exposure to the initial notes even further and produced even larger decrements in performance. These performance decrements were observed even though the final presentation in both the random and backward conditions included the same amount of information as the fixed condition. Thus, melody identification relied on early exposure to the initial notes of the tune.

The importance of the initial notes having been established, the secondary goal was to explain why initial segments of the melody are crucial for identification. Two hypotheses were considered. The structural centrality/ expectation hypothesis held that the initial notes of the melody help the listener perceive its global organization (key, meter, rhythm), which facilitates identification by enabling the listener to generate expectations about what notes will follow (Jones \& Boltz, 1989; Narmour, 1990). The data were consistent with this hypothesis in that the initial notes of the melody were more central to the global 
organization of the melody than were the initial notes heard in the backward condition. In contrast, the data did not support the serial organization/search hypothesis, which argued that initial notes are crucial for identification because the musical lexicon is indexed, and consequently searched, by the initial notes of the melody. According to this hypothesis, differences between the initial intervals in the fixed (or forward) and backward conditions should have predicted performance differences between the conditions; this relationship was not observed. However, the present data cannot completely dismiss the serial organization/search hypothesis because one could argue that semantic memory is organized in terms of the initial phrases rather than the initial intervals. This claim is supported by the high proportion of phrasing/meter errors observed in the confusion error analysis, and by previous work showing that interval-level information contributes little to melody identification (Schulkind et al., 2003).

The present data also have implications for theoretical explanations of PEI. Two major theories have been proposed to explain the effect. Competitive activation (Snodgrass \& Hirshman, 1991), which has been successful in accounting for a wide range of data using verbal and pictorial stimuli, relies on spreading activation. This account claims that activation of the target features present in highly degraded versions of the target automatically spreads to nontarget features and that the activation of these nontarget features is responsible for PEI. This model has difficulty explaining the present data because there is no reason to suspect that either random or backward presentation would activate more nontarget features than would forward presentation. The feature modulation search model (Schulkind, 2002) argues that melody identification results from a strategic search of semantic memory. Presenting the information in an unexpected order (e.g., the random and backward conditions) interfered with identification because it altered the region of memory space that was searched. Furthermore, the high proportion of phrasing/meter errors suggests that listeners use phrase-length chunks of information to search memory. This conclusion is also supported by low correlations between the initial intervals of various conditions and performance differences between those conditions. Presumably, when the amount of information initially available is highly constrained, listeners combine the available information with their knowledge of Western music to generate a plausible search cue.

This formulation of the feature modulation search model assumes that semantic memory for melodies is organized according to musical information contained in the initial phrase of the melody. Although proposing that musical semantic memory is organized musically borders on a tautology, illuminating the nature of the musical organization has proved difficult. For example, Halpern (1984) found that listeners could organize a set of familiar tunes more easily and coherently according to semantic charac- teristics like genre than they could according to musical characteristics like initial interval, mode, or rhythm. The large number of genre-based confusion errors observed in Experiment 2 is consistent with the pattern reported by Halpern and also supports the claim that conceptual information influences melody identification (Schulkind, 2004). That is, the data suggest that low-level perceptual information activated superordinate categorical information that the listener used to generate a response. However, the relatively high proportion of phrasing/meter errors contradicts Halpern's claim that semantic relationships are the predominant dimension along which musical knowledge is organized. The different interpretations reached by these two experiments may be due to methodological differences in stimulus presentation (music vs. index cards) and experimental task (sorting vs. identification) and must be interpreted cautiously because the total number of confusion errors was quite small $(N=83)$. Future work might increase the rate of incorrect guessing by employing an easier fixed condition, which might prompt more guesses by raising the subjects' overall confidence.

More generally, the importance of initial phrases for melody identification suggests a correspondence between melody and spoken word identification, and a separation of these two stimulus classes from identification of visual objects (see Kubovy \& Van Valkenburg, 2001, and Neuhoff, 2003, for a debate regarding similarities between visual and auditory "object" perception). Because words and melodies both possess strict linear structures (MarslenWilson, 1987; Marslen-Wilson \& Tyler, 1980), one would expect a verbal analogue of the backward condition to produce severe decrements in identification performance (although such an experiment would have to address coarticulation effects in spoken words). In contrast, visual stimuli would be much less likely to show significant effects of the order in which information was revealed because they are not organized along a temporal dimension.

Visual and nonvisual stimuli may also differ in terms of whether they are processed holistically or analytically (Banich \& Heller, 1998). Biederman (1987) argued that visual objects are analyzed piece by piece, or geon by geon, and has shown that highly degraded objects can still be identified if the remaining information allows the viewer to recapitulate the component geons. Laeng, Shah, and Kosslyn (1999) showed that the tendency to break an image down into its components is even more pronounced for unfamiliar images, such as those used in the PEI literature. In contrast, research on melody perception and identification suggests that melodies are frequently processed holistically, especially by novice listeners (Bever \& Chiarello, 1974; Gates \& Bradshaw, 1977a, 1977b; Peretz \& Morais, 1987; Peretz, Morais, \& Bertelson, 1987; Samson \& Zatorre, 1988; Schulkind et al., 2003; Zatorre, 1979, 1985). The holistic/analytic distinction may explain why incorrect hypotheses affect melody identification (Schulkind, 2002) but not picture identification (Snodgrass \& Hirshman, 1991). When trying to decide whether 
a highly degraded object is a "rutabaga," one can eliminate this possibility by identifying one geon that is not part of a "rutabaga." In contrast, when trying to decide if a highly degraded melody is "The Girl from Ipanema," one cannot rely on a single feature (i.e., a single note) in the melody because individual elements in a melody are interpreted in terms of the global structure or key. Therefore, one must maintain two global patterns in memory so that the physical stimulus can be compared with the stored representation of "The Girl from Ipanema." Thus, eliminating an incorrect melody title from consideration requires the listener to juggle more information simultaneously than is required to eliminate an incorrect visual object name from consideration.

In sum, the present experiments addressed two issues central to the field of music cognition: how melodies are identified and how musical semantic memory is organized. The data suggest that semantic memory is organized according to the musical structure of the initial phrases in the melody, but they are also consistent with earlier claims regarding the importance of conceptual, genre-based information (Halpern, 1984). The data also add to an emerging body of evidence demonstrating that melody identification relies on holistic processing that emphasizes the initial sounds of the melody. The importance of serial processing in melody identification also has implications beyond the music cognition literature because it suggests that the processes involved in identifying auditory-temporal stimuli differ from those involved in identifying visuospatial stimuli. Given the frequency with which people encounter auditory-temporal stimuli outside of the laboratory (e.g., familiar music, familiar voices, etc.), additional research should be devoted to specifying how these stimuli are represented in memory and subsequently identified.

\section{REFERENCES}

Banich, M. T., \& Heller, W. (1998). Evolving perspective on lateralization of function. Current Directions in Psychological Science, 7 , $1-2$.

Bartlett, J. C., \& Snelus, P. (1980). Lifespan memory for popular songs. American Journal of Psychology, 93, 551-560.

Bever, T. G., \& Chiarello, R. J. (1974). Cerebral dominance in musicians and nonmusicians. Science, 185, 537-539.

Biederman, I. (1987). Recognition-by-components: A theory of human image understanding. Psychological Review, 94, 115-147.

BRUner, J. S., \& PotTer, M. C. (1964). Interference in visual recognition. Science, 144, 424-425.

BUTLER, D. (1992). The musician's guide to perception and cognition. New York: Macmillan.

CudDy, L. L. (1993). Melody comprehension and tonal structure. In T. J. Tighe \& W. J. Dowling (Eds.), Psychology and music: The understanding of melody and rhythm (pp. 19-38). Hillsdale, NJ: Erlbaum.

DowLING, W. J. (1974). The perception of interleaved melodies. Cognitive Psychology, 5, 322-337.

Dowling, W. J., LUNG, K.-T. M., \& Herrbold, S. (1987). Aiming attention in pitch and time in the perception of interleaved melodies. Perception \& Psychophysics, 41, 642-656.

Gates, A., \& BradshaW, J. L. (1977a). Music perception and cerebral asymmetries. Cortex, 13, 390-401.

Gates, A., \& BRADShaW, J. L. (1977b). The role of the cerebral hemispheres in music. Brain \& Language, 4, 403-431.
Gibson, J. M., \& WatKins, M. J. (1991). An auditory cue-depreciation effect. American Journal of Psychology, 104, 439-446.

HalPern, A. R. (1984). Organization in memory for familiar songs. Journal of Experimental Psychology: Learning, Memory, \& Cognition, 10, 496-512.

HÉbert, S., \& Peretz, I. (1997). Recognition of music in long-term memory: Are melodic and temporal patterns equal partners? Memory \& Cognition, 25, 518-533.

JoNES, M. R., \& BoLTZ, M. (1989). Dynamic attending and reactions to time. Psychological Review, 96, 459-491.

JusczyK, P. W., \& LuCE, P. A. (2002). Speech perception. In H. Pashler \& S. Yantis (Eds.), Stevens' Handbook of experimental psychology: Vol. 1. Sensation and perception (3rd ed., pp. 493-536). New York: Wiley.

KrumhansL, C. L. (1979). The psychological representation of musical pitch in a tonal context. Cognitive Psychology, 11, 346-374.

Krumhansl, C. L. (1990). Cognitive foundations of musical pitch. New York: Oxford University Press.

Kubovy, M., \& VAN VALKenburG, D. (2001). Auditory and visual objects. Cognition, 80, 97-126.

LAENG, B., SHAH, J., \& Kosslyn, S. (1999). Identifying objects in conventional and contorted poses: Contributions of hemisphere-specific mechanisms. Cognition, 70, 53-85.

LiNDFIELD, K. C., \& WingFIELD, A. (1999). An experimental and computational analysis of age differences in the recognition of fragmented pictures: Inhibitory connections versus speed of processing. Experimental Aging Research, 25, 223-242.

LiNDFIELD, K. C., WiNGFiELD, A., \& Bowles, N. L. (1994). Identification of fragmented pictures under ascending versus fixed presentation in young and elderly adults: Evidence for the inhibition-deficit hypothesis. Aging \& Cognition, 4, 1-10.

Luo, C. R., \& SNodgrass, J. G. (1994). Competitive activation model of perceptual interference in picture and word identification. Journal of Experimental Psychology: Human Perception \& Performance, $\mathbf{2 0}_{2}$ 50-60.

MARSLEN-WiLson, W. D. (1987). Functional parallelism in spoken word-recognition. Cognition, 25, 71-102.

Marslen-Wilson, W. [D.], Moss, H. E., \& van Halen, S. (1996). Perceptual distance and competition in lexical access. Journal of Experimental Psychology: Human Perception \& Performance, 22, 13761392

Marslen-Wilson, W. [D.], \& Tyler, L. K. (1980). The temporal structure of spoken language understanding. Cognition, 8, 1-71.

Marslen-Wilson, W. [D.], \& ZWitserloOD, P. (1989). Accessing spoken words: The importance of word onsets. Journal of Experimental Psychology: Human Perception \& Performance, 15, 576-585.

NARMOUR, E. (1990). The analysis and cognition of basic melodic structures: The implication-realization model. Chicago: University of Chicago Press.

Neuhoff, J. G. (2003). Pitch variation is unnecessary (and sometimes insufficient) for the formation of auditory objects. Cognition, 87, 219224.

Peretz, I., \& Morais, J. (1987). Analytic processing in the classification of melodies as same or different. Neuropsychologia, 25, 645-652.

Peretz, I., Morais, J., \& Bertelson, P. (1987). Shifting ear differences in melody recognition through strategy inducement. Brain \& Cognition, 6, 202-215.

PeynircioĞLU, Z. F. (1987). Inhibition through incremental fragment cuing with primed items. Journal of Experimental Psychology: Learning, Memory, \& Cognition, 13, 569-572.

PEYNIRCIOǦLU, Z. F. (1990). Trying harder may disrupt cue utilisation. European Journal of Cognitive Psychology, 2, 363-373.

PeynircioĞLu, Z. F., \& Watkins, M. J. (1986). Cue-depreciation: When word fragment completion is undermined by prior exposure to lesser fragments. Journal of Experimental Psychology: Learning, Memory, \& Cognition, 12, 426-431.

SAMSON, S., \& ZATORRE, R. J. (1988). Melodic and harmonic discrimination following unilateral cerebral excision. Brain \& Cognition, 7, 348-360.

SCHULKIND, M. D. (2000). Perceptual interference decays over short unfilled intervals. Memory \& Cognition, 28, 949-956.

SCHULKIND, M. D. (2002). Feature modulation search: A novel memory 
search model that extends the perceptual interference effect to musical stimuli. Journal of Experimental Psychology: Learning, Memory, \& Cognition, 28, 346-352.

SCHULKIND, M. D. (2004). Conceptual and perceptual information both influence melody identification. Memory \& Cognition, 32, 841-851.

Schulkind, M. D., Hennis, L. K., \& Rubin, D. C. (1999). Music, emotion, and autobiographical memory: They're playing your song. Memory \& Cognition, 27, 948-955.

Schulkind, M. D., Shulman, R. J., \& Rubin, D. C. (2003). Musical features that facilitate melody identification: How do you know it's "your" song when they finally play it? Music Perception, 21, 217249.

Smith, J. D., Kemler Nelson, D. G., \& Appleton, T. (1994). What child is this? What interval was that? Familiar tunes and music perception in novice listeners. Cognition, 52, 23-54.

SNODGRASS, J. G., \& Hirshman, E. (1991). Theoretical explorations of the Bruner-Potter (1964) interference effect. Journal of Verbal Learning \& Verbal Behavior, 30, 273-293.

Warren, P., \& Marslen-Wilson, W. [D.] (1987). Continuous uptake of acoustic cues in spoken word recognition. Perception \& Psychophysics, 41, 262-275.

WARREN, P., \& MARSLEN-WiLSON, W. [D.] (1988). Cues to lexical choice: Discriminating place and voice. Perception \& Psychophysics, 43, 21-30.

White, B. W. (1960). Recognition of distorted melodies. American Journal of Psychology, 73, 100-107.

YALCH, R. F. (1991). Memory in a jingle jungle: Music as a mnemonic device in communicating advertising slogans. Journal of Applied Psychology, 76, 268-275.

ZATORRE, R. J. (1979). Recognition of dichotic melodies by musicians and nonmusicians. Neuropsychologia, 17, 607-617.

ZATORRE, R. J. (1985). Discrimination and recognition of tonal melodies after unilateral cerebral excisions. Neuropsychologia, 23, 31-41. 
APPENDIX

\begin{tabular}{ll}
\hline \multicolumn{1}{c}{ Correct Title } & Conceptual Similarity \\
\hline Amazing Grace & Taps (2) \\
America, the Beautiful & National Anthem \\
Clap Your Hands & $\begin{array}{c}\text { She'll be Coming Around } \\
\text { the Mountain }\end{array}$
\end{tabular}

Clementine

Phrasing/Meter

Over There

$\mathrm{O}$, Christmas Tree

Lullabye and Good Night

O, Come All Ye Faithful

Saints Go Marching In

Here Comes the Bride In the Navy

O, Christmas Tree

Dixie

Do-Re-Mi

Frère Jacques

Frosty

Happy Birthday

Home on the Range

Itsy-Bitsy Spider

I've Been

Working...Railroad

Joy to the World

Lean on $\mathrm{Me}$

Stand by Me

London Bridge

Mary Had a Little Lamb

Jack \& Jill

Rudolph (3)

Santa Claus is Coming to Town

Little Bunny FooFoo

London Bridge

Do-Re-Mi (2)

Indiana Jones theme

National Anthem

It's a Small World

Alouette

O, Christmas Tree

She'll be Coming Around the Mountain

Here Comes the Bride (2)

Yankee Doodle (3)

London Bridge (2)

Away in a Manger

London Bridge

Let's Go Fly a Kite

Rudolph

Up on the House Top

Over the Rainbow

My Bonnie Lies... Ocean

Bingo

Marine Corps Hymn (3)

Over There

Hot Crossed Buns (4)

This Old Man

Mary Had a Little Lamb (2)

Yankee Doodle

Comedy Tonight

Battle Hymn of the Republic

Saints Go Marching In

Heart and Soul

Saints Go Marching In

Here Comes the Bride Saints Go Marching In

Yankee Doodle

Pop! Goes the Weasel

Yankee Doodle

Rudolph

Oh, Susannah

Yankee Doodle

Yankee Doodle

Dixie

Figaro

See Where She Goes

Frosty (2)

It's a Small World

Three Blind Mice

God Rest Y...Gentlemen

Doogie Howser Theme

White Christmas

When You Wish Upon a Star

Yankee Doodle

Old McDonald (2)

London Bridge

Yesterday

Total 